ISSN 2073-4409

www.mdpi.com/journal/cells

Review

\title{
Ciliary/Flagellar Protein Ubiquitination
}

\section{Huan Long, Qiyu Wang and Kaiyao Huang *}

Key Laboratory of Algal Biology, Institute of Hydrobiology, Chinese Academy of Sciences, Wuhan 430072, China; E-Mails: huanlong@ihb.ac.cn (H.L.); ihbwqy2015@gmail.com (Q.W.)

* Author to whom correspondence should be addressed; E-Mail: huangky@ihb.ac.cn; Tel.:+86-27-68780901.

Academic Editors: William Tsang and Gang Dong

Received: 7 June 2015 / Accepted: 28 August 2015 / Published: 2 September 2015

\begin{abstract}
Cilia/flagella are conserved eukaryotic organelles that play an important role in the control of cell motility and detection of environmental cues. However, the molecular mechanisms underlying ciliary/flagellar assembly, maintenance, disassembly, and signal transduction are not yet completely understood. Recent studies demonstrated that post-translational modifications (PTMs) such as phosphorylation, methylation, glutamylation, and ubiquitination are involved in these processes. In this mini review, we present a summary of research progress in ciliary/flagellar protein ubiquitination, including the ubiquitin conjugation system identified by proteomics as well as the role of ciliary/flagellar protein ubiquitination in flagellar disassembly, motility, and signal transduction. Moreover, we described putative further research directions in the study of ciliary/flagellar protein ubiquitination.
\end{abstract}

Keywords: flagella; ubiquitination; post-translational modifications; sperm; primary cilia

\section{Introduction}

Cilia/flagella are microtubule-based organelles projecting from the surface of most eukaryotic cells (cilia and flagella are used interchangeably, this review only refers to eukaryotic flagella, not bacterial and archaeal flagella). Cilia are composed of basal body, axoneme (nine triplets of microtubules as the core structure), and the ciliary membrane (Figure 1A). Most motile cilia possess a $9+2$ structure, in which the axoneme possesses a central pair of microtubules (Figure 1B). However, immotile primary 
cilia have a $9+0$ structure (Figure 1C). The main functions of cilia include controlling cell motility, sensing environmental cues, and mediating ciliary signal transduction [1-5]. These functions depend on the homeostasis of the length and the composition of cilia/flagella [1-5]. Ciliary/flagellar length is the result of a balance between assembly and disassembly (turnover) of microtubules at the tip. Since no protein synthesis occurs within the cilia/flagella, all axonemal precursors and turnover products need to be transported via Intraflagellar Transport (IFT), which is critical not only for flagellar assembly and disassembly but also for mediating signal transduction in cilia. Mutations in the IFT system and other conserved ciliary proteins directly result in ciliopathies, such as Polycystic Kidney Disease, repeated chest infections, congenital heart disease, male infertility, blindness, and obesity [6-10].
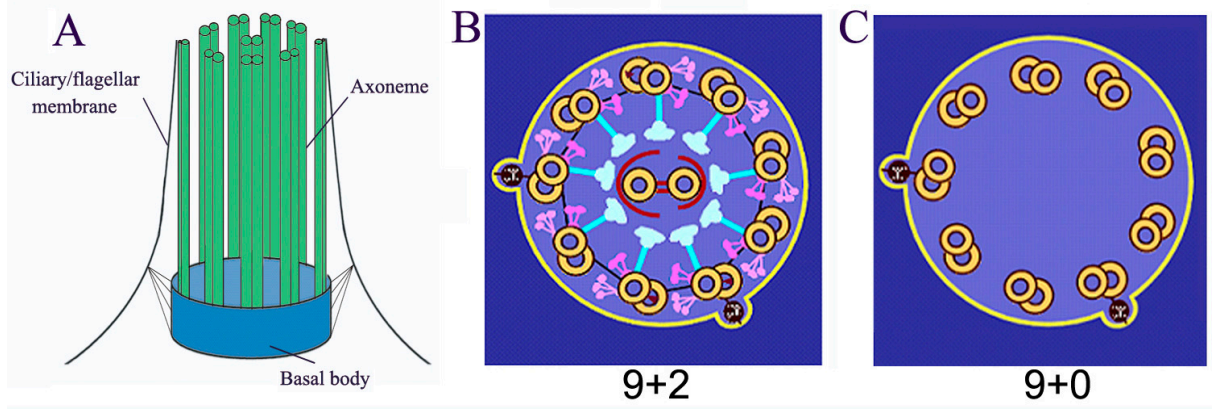

Figure 1. Basal structure of cilia/flagella. (A) Cilia/flagella are composed of axoneme, ciliary/flagellar membrane and basal body; (B) "9 + 2" motile cilia/flagella possess a central pair and 9 triple microtubule with associated protein complexes such as outer dynein, inner dynein, and radial spoke; (C) “9 + 0" immotile cilia/flagella only have 9 triple microtubule.

Cilia/flagella are polarized organelles and their assembly/disassembly is highly regulated. Flagella assemble when cells exit from the cell cycle and enter into the stationary phase (G0), while they disassemble prior to the mitosis phase of the cell cycle. The processes of ciliary assembly and disassembly are complex, involving processes such as gene expression, protein transport, and vesicle trafficking. One of the key steps is selective transport. Anterograde IFT interacts with axonemal precursors at the base and transports them to the tips of the cilia, whereas retrograde IFT loads turnover products at the tip and transport them to the base [5,11]. Recent studies demonstrated that various types of PTMs (post-translational modifications) such as phosphorylation, methylation, glutamylation, and ubiquitination are involved in these highly regulated processes. For instance, microtubules undergo acetylation, glycylation, glutamylation, and ubiquitination [12-15].

The functions of protein phosphorylation in regulating flagellar assembly, disassembly, and length control are well documented [16]. Flagellar assembly requires glycogen synthase kinase (GSK)-3 activity and inhibition of GSK-3 $\beta$ results in short flagella [17]. Phosphorylation of Chlamydomonas aurora-like kinase (CALK) is a marker of flagellar length during both assembly and disassembly [18]. The flagellar-shortening mutant FLS1 encodes a cyclin-dependent kinase-like kinase, which regulates the phosphorylation of CALK and CrKinesin13; both these kinases are required for disassembly of the distal portion of Chlamydomonas flagella [19]. In addition, one specific proteomic study reported that 89 different phosphoproteins have been identified in the process of flagella shortening, indicating dramatic changes in protein phosphorylation during flagellar disassembly [20]. 
Methylation is another form of PTM involved in flagellar assembly and disassembly. Schneider et al., first showed that flagella contain a cobalamin-independent form of methionine synthase (MetE), whose amount is lowest in full-length flagella, increases in assembling flagella, and is highest in disassembling flagella [21]. In addition, glutamylation may also play a role in flagellar motility. Tubulin can be polyglutamylated by the tubulin tyrosine ligase like protein (TTLL) family, and tubulin glutamylation is essential for regulation of beating asymmetry [22].

To date, ubiquitin has been found only in eukaryotic organisms. Ubiquitination is responsible for controlling an extensive range of physiological processes such as appropriate cell cycle progression, transcriptional regulation, protein quality control, signal transduction, membrane protein endocytosis, intracellular trafficking, and DNA repair [23]. Ubiquitination is a form of PTMs in which ubiquitin molecules are covalently attached to target proteins by a series of enzymes including ubiquitin-activating enzyme (E1), ubiquitin conjugating enzyme (E2), and ubiquitin ligase (E3) $[23,24]$. Polyubiquitin chains are generated via an isopeptide bond formed between a carboxyl-terminal glycine of one ubiquitin (G76) and a specific lysine residue of another ubiquitin (i.e., K6, K11, K27, K29, K33, $\mathrm{K} 48$, or K63). K48-linked ubiquitination generally functions as a signal for degradation in the $26 \mathrm{~S}$ proteasome, whereas K63-linked ubiquitination has non-proteolytic functions such as membrane protein trafficking, endocytosis, and DNA repair [23,25]. Monoubiquitin and the K63 chain can be removed from the targets by deubiquitylating enzymes, making these modifications reversible [23].

The majority of proteins with K48-linked ubiquitin chains are degraded by the ubiquitin-proteasome system. When a substrate is coupled with this polyubiquitin chain, it binds to the $26 \mathrm{~S}$ proteasome complex, in which the substrate is unfolded, cleaved, and degraded into small peptides and then the ubiquitin is recycled [23,26].

Recently, protein ubiquitination was found in different cilia/flagella such as flagella of Chlamydomonas, sperm flagella and primary cilia. Ubiquitination is involved in regulation of ciliary assembly and disassembly, signal transduction, spermatogenesis, or ciliogenesis. In this mini-review, we focus on ciliary/flagellar protein ubiquitination and discuss its putative functions.

\section{Ubiquitin Conjugating System is Associated with Cilia}

The protein of ubiquitin conjugating system was first found in the flagellar proteomes of Chlamydomonas [27], which is an excellent organism to study flagella. It has well-established genetics. The flagella of Chlamydomonas are easily isolated, and the genome sequence and proteomic data from both the flagella and basal body are available [27,28]. The Chlamydomonas flagellar proteome includes a single E1-activating enzyme, four E2-conjugating enzymes, and three E3-ligases [27-29]. In addition, the ubiquitin conjugating system proteins were also found in proteomes of primary cilia and mouse photoreceptor sensory cilium [30,31]. For instance, ubiquitin, ubiquitin-like protein, a single E1 activating enzyme, four E2 conjugating enzymes, and two E3 ligases had been identified in the proteome of the mouse photoreceptor sensory cilium [30]. Thus, the ubiquitin conjugation system is associated with motile cilia and primary cilia.

Direct evidence of the association of ubiquitin conjugation system with flagella was obtained from an in vitro flagellar ubiquitination experiment [15]. Human influenza hemagglutinin (HA) labeled ubiquitin was added to isolated flagella, and in the presence of ATP, HA-labeled ubiquitin was 
conjugated with several flagellar proteins. This result demonstrated that not only the all three types of enzymes of the ubiquitin-conjugation system located in flagella but also they can function together to add ubiquitins to flagellar proteins.

\section{Flagellar Protein Ubiquitination and Flagellar Disassembly}

The activity of ubiquitin conjugation system can also be determined using the in vitro flagellar conjugation system. The ubiquitin conjugation activity in flagella was higher when using shortening flagella compared to static flagella, which suggested that ubiquitination is related to flagellar disassembly [15]. Indeed, the pattern of ubiquitinated proteins is similar in assembling flagella as compared to static flagella; however, flagellar ubiquitinated proteins increased during chemically induced flagellar disassembly or naturally occurring flagellar resorption [15]. Furthermore, more ubiquitinated proteins were accumulated in flagella when IFT is blocked, especially in flagella from mutants with defects in retrograde IFT. Since no subunit of the proteasome was found in the Chlamydomonas flagellar proteome [27], according to this result, ubiquitinated proteins need to be transported to the cell body. Hence, a working model of the ubiquitin-conjugation system in flagellar disassembly was proposed (Figure 2). The turnover products were labeled with ubiquitin and ubiquitinated proteins were transported to the cell body via retrograde IFT. During flagella shortening induced by NaPPi in wild-type cells, ubiquitinated proteins are transported to the cell body via IFT; therefore, small amounts of ubiquitinated proteins were detected in the shortening flagella. When IFT was blocked in fla 10 at a restrictive temperature or in retrograde IFT mutants, the ubiquitinated proteins could not be transported to the cell body and a large amount of ubiquitinated proteins accumulated in flagella [15]. However, the detailed mechanism needs to be clarified by further studies.

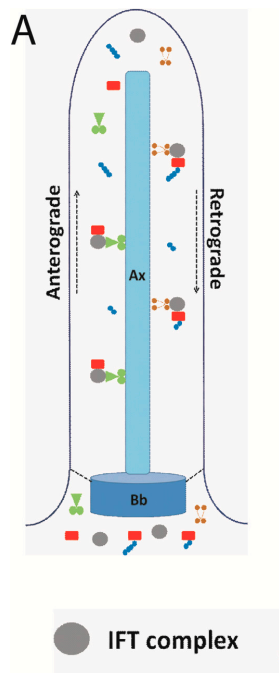

B
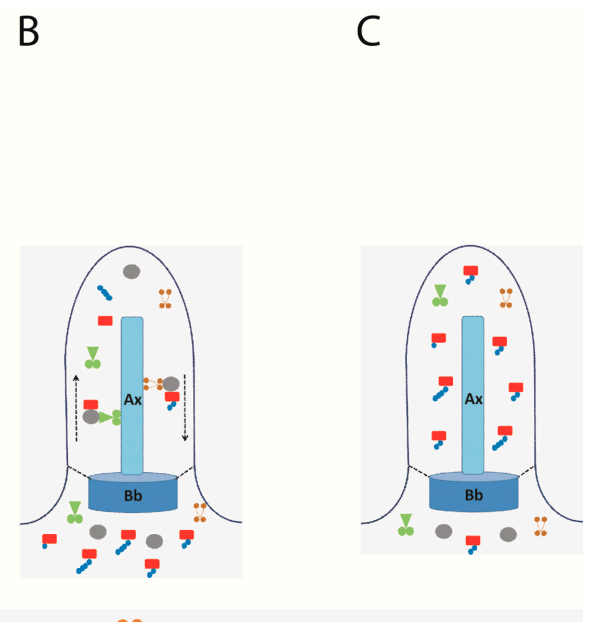

Kinesin- II Dynein

Cargo

Ubiquitin

Figure 2. A working model of IFT transporting the ubiquitinated proteins. (A) IFT complex moves bi-directionally underneath the flagellar membrane; (B) During flagellar disassembly, the turnover products were ubiquitinated and transported to the cell body by retrograde IFT; (C) When IFT stops or in retrograde IFT mutants, the ubiquitinated proteins accumulated in the flagella. Ax: Axoneme, Bb: Basal body, dash line indicated transition fiber, solid line indicated flagellar membrane; arrowheads indicated the move direction of anterograde IFT and retrograde IFT. 


\section{Flagellar Protein Ubiquitination and Signal Transduction}

The ubiquitination may also play a role in regulating signal transduction in flagella. The cation channel $\mathrm{CrPKD}_{2}$ and kinase $\mathrm{CrPKG}$, both of which are components of a cAMP-dependent signal transduction pathway in mating, can be ubiquitinated in flagella [15]. The amount of the ubiquitin-conjugating enzyme CrUbc13 also increased rapidly in the flagella after mating and some flagellar proteins were ubiquitinated within 5 min of mixing of the plus and minus gametes [15]. These data suggested that the ubiquitin-conjugating system is not only involved in the regulation of flagellar length but might also participate in regulating flagellar signal transduction [15].

\section{Flagellar Protein Ubiquitination and Spermatogenesis}

Spermatogenesis is the process by which spermatogenic stem cells undergo mitotic and meiotic division and differentiate to streamlined spermatozoa which are capable of motility and fertilization [32]. One of the key events in spermatogenesis is the formation of the flagella, which enables sperm to reach eggs for fertilization. The axoneme, consisting of a conserved central microtubule pair and nine outer microtubule doublets, is the core structure of sperm flagella, and is assembled soon after meiosis [32]. The sperm axoneme is extensively post-translationally modified, including acetylation, tyrosination, and ubiquitination [33-35].

The 26S proteasome complex mediating the degradation of polyubiquitinated proteins was isolated from the rat testis and sperm tail in 2000; this finding linked ubiquitination with spermatogenesis [36]. Subsequent work confirmed that the ubiquitin-proteasome system plays a crucial role in spermatogenesis. For example, the RNF8 E3 enzyme mediates histone ubiquitination, which facilitates histone replacement in elongating spermatids [37]. In mammals, the membrane-associated, Really Interesting New Gene (RING)-CH (MARCH) family comprises 11 RING finger-containing E3 ubiquitin ligases. Of these, MARCH7, MARCH10, and MARCH11 are highly expressed in developing spermatids. MARCH7 is localized to the acroplaxome and flagella and may play a role in the regulation of head shaping and flagellar formation [38]. MARCH10 is localized in the fibrous sheath within the principal piece of the flagella and may be involved in flagellar assembly and maintenance in elongating spermatids [39]. MARCH11 may regulate the transport of transmembrane glycoproteins between the trans-Golgi network and multivesicular bodies in round spermatids [40,41]. In addition, aberrant head morphology has been observed in spermatozoa of mice lacking the UBE2B E2 enzyme [42]. Male mice lacking the TATA element modulatory factor/androgen receptor co-activator 160 (TMF/ARA160) E3 enzyme are sterile due to abnormal sperm development such as acrosome disruption and coiled flagella $[43,44]$. These data suggested that ubiquitination plays a critical role in spermatogenesis.

\section{Ciliary Protein Ubiquitination in Primary Cilia}

The primary cilium functions as an antenna of the cell, protruding directly from the distal end of mother centriole (basal body). The ubiquitin-proteasome system is necessary to initiate ciliogenesis by removing the keratin-binding protein trichoplein from the mother centrioles. Trichoplein is polyubiquitinated at Lys-50 and Lys-57 by Cul3-RING ubiquitin ligase. Degradation of polyubiquitinated trichoplein plays an essential role in the initial step of axonemal extension during 
ciliogenesis [45]. The E3 ubiquitin ligase Mindbomb 1 (MIB1) is a new component of centriolar satellites; it ubiquitinates Cep131 (also known as AZI1) and pericentriolar material 1 (PCM1) and suppresses primary cilium formation [46]. Taken together, ubiquitination occurs during both initiation and elongation stages of ciliogenesis.

Ubiquitination can function antagonistically or synergistically with other PTMs. For example, tumor suppressor Von Hippel-Lindau protein (VHL) is also an E3 ligase, which stabilizes microtubules in the primary cilium. The kinase Nek1 phosphorylates VHL at multiple sites and promotes its ubiquitination and degradation in the proteasome. Mutation of VHL at serine-168, a Nek1-phosphorylation site, increases the stability of VHL and improves its capacity to stabilize cilia [47]. Apart from protein ubiquitination, protein deubiquitination also plays a role in ciliogenesis. Cylindromatosis tumor suppressor gene $(C Y L D)$ encodes a deubiquitinating enzyme that removes Lys63- or linear-linked ubiquitin chains, and $C Y L D$ mutation results in defects of cilium formation [48]. Ubiquitin-specific protease (USP)- 8 functions as a deubiquitinating enzyme of Hypoxia-Inducible Factor-1 $\alpha$ (HIF1 $\alpha$ ) by counteracting the Hippel-Lindau (pVHL)-mediated ubiquitination of HIF1 $\alpha$, and maintains basal expression level of HIF1 $\alpha$, which is critical for endosome trafficking-mediated ciliogenesis [48].

\section{Summary and Future Research Directions}

Considerable progress has been made in the determination of the functions of ubiquitination in flagellar disassembly, flagellar signal transduction, spermatogenesis, and ciliogenesis. Ubiquitination is a conserved form of PTM that associates with both motile and primary cilia. However, several questions regarding ciliary/flagellar ubiquitination still need to be addressed: First, whether the ubiquitin conjugation system also presents in primary cilia, right now we only have the support evidence from the proteomic analysis [31]. Second, what are the substrates of ciliary ubiquitination when cilia undergo assembly, disassembly and how a variety of mono ubiquitin or poly ubiquitin chains are added to each substrate? In addition, the E1, E2, and substrate specific E3 need to be identified for each substrate. Third, what is the final fate of ciliary ubiquitinated proteins, reutilized or degraded in proteasome of cell body? Fourth, how to determine the specific functions of protein ubiquitination in cilia/flagella taking into account the fact that flagellar E1-activating enzyme and other components of the ubiquitin conjugation system are also found in the cell body. Fifth, how the ciliary ubiquitination events are regulated, the signal is from the cilia or from the cell body. Clearly, several ciliary research models and new technologies need to be combined to explore these questions. Addressing these gaps will further facilitate our understanding of the function of ubiquitination in cilia/flagella.

\section{Acknowledgments}

This work was supported in part by the National Nature Science Foundation of China (Grant 31171287 to Huang K. and 31401154 to Long H.).

\section{Author Contributions}

Kaiyao Huang and Huan Long conceived and wrote the manuscript. Qiyu Wang participated in the design of the working model in Figure 2. All authors read and approved the final manuscript. 


\section{Conflicts of Interest}

The authors declare no conflict of interest.

\section{References}

1. Goetz, S.C.; Anderson, K.V. The primary cilium: A signalling centre during vertebrate development. Nat. Rev. Genet. 2010, 11, 331-344.

2. Shah, A.S.; Ben-Shahar, Y.; Moninger, T.O.; Kline, J.N.; Welsh, M.J. Motile cilia of human airway epithelia are chemosensory. Science 2009, 325, 1131-1134.

3. Pazour, G.J.; Witman, G.B. The vertebrate primary cilium is a sensory organelle. Curr. Opin. Cell Biol. 2003, 15, 105-110.

4. Singla, V.; Reiter, J.F. The primary cilium as the cell's antenna: Signaling at a sensory organelle. Science 2006, 313, 629-633.

5. Rosenbaum, J.L.; Witman, G.B. Intraflagellar transport. Nature reviews. Nat. Rev. Mol. Cell Biol. 2002, 3, 813-825.

6. Tilley, A.E.; Walters, M.S.; Shaykhiev, R.; Crystal, R.G. Cilia dysfunction in lung disease. Annu. Rev. Physiol. 2015, 77, 379-406.

7. Edwards, J.K. Polycystic kidney disease: Pathogenic missense mutations result in defective trafficking of polycystin-1 to cilia. Nat. Rev. Nephrol. 2015, 11, 4, doi:10.1038/nrneph.2014.222.

8. Li, Y.; Klena, N.T.; Gabriel, G.C.; Liu, X.; Kim, A.J.; Lemke, K.; Chen, Y.; Chatterjee, B.; Devine, W.; Damerla, R.R.; et al. Global genetic analysis in mice unveils central role for cilia in congenital heart disease. Nature 2015, 521, 520-524.

9. Dell, K.M. The role of cilia in the pathogenesis of cystic kidney disease. Curr. Opin. Pediatr. 2015, 27, 212-218.

10. Hildebrandt, F.; Benzing, T.; Katsanis, N. Ciliopathies. N. Engl. J. Med. 2011, 364, 1533-1543.

11. Dentler, W. Intraflagellar transport (IFT) during assembly and disassembly of Chlamydomonas flagella. J. Cell Biol. 2005, 170, 649-659.

12. Kubo, T.; Yanagisawa, H.A.; Liu, Z.; Shibuya, R.; Hirono, M.; Kamiya, R. A conserved flagella-associated protein in Chlamydomonas, FAP234, is essential for axonemal localization of tubulin polyglutamylase TTLL9. Mol. Biol. Cell 2014, 25, 107-117.

13. Kozminski, K.G.; Diener, D.R.; Rosenbaum, J.L. High level expression of nonacetylatable alpha-tubulin in Chlamydomonas reinhardtii. Cell Motil. Cytoskel. 1993, 25, 158-170.

14. Dossou, S.J.; Bre, M.H.; Hallworth, R. Mammalian cilia function is independent of the polymeric state of tubulin glycylation. Cell Motil. Cytoskel. 2007, 64, 847-855.

15. Huang, K.; Diener, D.R.; Rosenbaum, J.L. The ubiquitin conjugation system is involved in the disassembly of cilia and flagella. J. Cell Biol. 2009, 186, 601-613.

16. Cao, M.; Li, G.; Pan, J. Regulation of cilia assembly, disassembly, and length by protein phosphorylation. Methods Cell Biol. 2009, 94, 333-346.

17. Wilson, N.F.; Lefebvre, P.A. Regulation of flagellar assembly by glycogen synthase kinase 3 in Chlamydomonas reinhardtii. Eukaryot. Cell 2004, 3, 1307-1319. 
18. Cao, M.; Meng, D.; Wang, L.; Bei, S.; Snell, W.J.; Pan, J. Activation loop phosphorylation of a protein kinase is a molecular marker of organelle size that dynamically reports flagellar length. Proc. Natl. Acad. Sci. USA 2013, 110, 12337-12342.

19. Hu, Z.; Liang, Y.; He, W.; Pan, J. Cilia disassembly with two distinct phases of regulation. Cell Rep. 2015, 10, 1803-1810.

20. Pan, J.; Naumann-Busch, B.; Wang, L.; Specht, M.; Scholz, M.; Trompelt, K.; Hippler, M. Protein phosphorylation is a key event of flagellar disassembly revealed by analysis of flagellar phosphoproteins during flagellar shortening in Chlamydomonas. J. Proteome Res. 2011, 10, 3830-3839.

21. Schneider, M.J.; Ulland, M.; Sloboda, R.D. A protein methylation pathway in Chlamydomonas flagella is active during flagellar resorption. Mol. Biol. Cell 2008, 19, 4319-4327.

22. Ikegami, K.; Sato, S.; Nakamura, K.; Ostrowski, L.E.; Setou, M. Tubulin polyglutamylation is essential for airway ciliary function through the regulation of beating asymmetry. Proc. Natl. Acad. Sci. USA 2010, 107, 10490-10495.

23. Hochstrasser, M. Origin and function of ubiquitin-like proteins. Nature 2009, 458, 422-429.

24. Corbit, K.C.; Aanstad, P.; Singla, V.; Norman, A.R.; Stainier, D.Y.; Reiter, J.F. Vertebrate Smoothened functions at the primary cilium. Nature 2005, 437, 1018-1021.

25. Welchman, R.L.; Gordon, C.; Mayer, R.J. Ubiquitin and ubiquitin-like proteins as multifunctional signals. Nat. Rev. Mol. Cell Biol. 2005, 6, 599-609.

26. Ciechanover, A.; Kwon, Y.T. Degradation of misfolded proteins in neurodegenerative diseases: Therapeutic targets and strategies. Exp. Mol. Med. 2015, 47, e147.

27. Pazour, G.J.; Agrin, N.; Leszyk, J.; Witman, G.B. Proteomic analysis of a eukaryotic cilium. J. Cell Biol. 2005, 170, 103-113.

28. Arnaiz, O.; Malinowska, A.; Klotz, C.; Sperling, L.; Dadlez, M.; Koll, F.; Cohen, J. Cildb: A knowledgebase for centrosomes and cilia. Database (Oxford) 2009, 2009, bap022, doi:10.1093/database/bap022.

29. Long, H.; Huang, K. Analysis of flagellar protein ubiquitination. Methods Enzymol. 2013, 524, 59-73.

30. Liu, Q.; Tan, G.; Levenkova, N.; Li, T.; Pugh, E.N., Jr.; Rux, J.J.; Speicher, D.W.; Pierce, E.A. The proteome of the mouse photoreceptor sensory cilium complex. Mol. Cell. Proteomics 2007, 6, 1299-1317.

31. Ishikawa, H.; Thompson, J.; Yates, J.R., 3rd; Marshall, W.F. Proteomic analysis of mammalian primary cilia. Curr. Biol. 2012, 22, 414-419.

32. O’Donnell, L.; O’Bryan, M.K. Microtubules and spermatogenesis. Semin. Cell Dev. Biol. 2014, $30,45-54$.

33. Hermo, L.; Pelletier, R.M.; Cyr, D.G.; Smith, C.E. Surfing the wave, cycle, life history, and genes/proteins expressed by testicular germ cells. Part 3: Developmental changes in spermatid flagellum and cytoplasmic droplet and interaction of sperm with the zona pellucida and egg plasma membrane. Microsc. Res. Tech. 2010, 73, 320-363.

34. Kierszenbaum, A.L. Sperm axoneme: A tale of tubulin posttranslation diversity. Mol. Reprod. Dev. 2002, 62, 1-3.

35. Bose, R.; Manku, G.; Culty, M.; Wing, S.S. Ubiquitin-proteasome system in spermatogenesis. Adv. Exp. Med. Biol. 2014, 759, 181-213. 
36. Mochida, K.; Tres, L.L.; Kierszenbaum, A.L. Structural features of the 26S proteasome complex isolated from rat testis and sperm tail. Mol. Reprod. Dev. 2000, 57, 176-184.

37. Markelewicz, R.J., Jr.; Hall, S.J.; Boekelheide, K. 2,5-Hexanedione and carbendazim coexposure synergistically disrupts rat spermatogenesis despite opposing molecular effects on microtubules. Toxicol. Sci. 2004, 80, 92-100.

38. Zhao, B.; Ito, K.; Iyengar, P.V.; Hirose, S.; Nakamura, N. MARCH7 E3 ubiquitin ligase is highly expressed in developing spermatids of rats and its possible involvement in head and tail formation. Histochem. Cell Biol. 2013, 139, 447-460.

39. Iyengar, P.V.; Hirota, T.; Hirose, S.; Nakamura, N. Membrane-associated RING-CH 10 (MARCH10 protein) is a microtubule-associated E3 ubiquitin ligase of the spermatid flagella. J. Biol. Chem. 2011, 286, 39082-39090.

40. Yasuzumi, G.; Matano, Y.; Asai, T.; Nagasaka, M.; Yasuzumi, F. Spermatogenesis in animals as revealed by electron microscopy. XXII. Development of nuclei and cytoplasmic microtubules in the grasshopper spermatids. Z. Zellforsch. Mikrosk. Anat. 1971, 115, 543-552.

41. Yasuzumi, G.; Sugioka, T. Spermatogenesis in animals as revealed by electron microscopy. XXI. Microkaryosomes and microtubules appearing during spermiogenesis of the lovebird Uroloncha striata var. domestica flower. Z. Zellforsch. Mikrosk. Anat. 1971, 114, 451-459.

42. Fouquet, J.; Kann, M.; Soues, S.; Melki, R. ARP1 in Golgi organisation and attachment of manchette microtubules to the nucleus during mammalian spermatogenesis. J. Cell Sci. 2000, 113, 877-886.

43. Lerer-Goldshtein, T.; Bel, S.; Shpungin, S.; Pery, E.; Motro, B.; Goldstein, R.S.; Bar-Sheshet, S.I.; Breitbart, H.; Nir, U. TMF/ARA160: A key regulator of sperm development. Dev. Biol. 2010, 348, 12-21.

44. Ugwunna, S.C.; Foor, W.E. The function of microtubules during spermatogenesis of Ancylostoma caninum. J. Parasitol. 1982, 68, 817-823.

45. Kasahara, K.; Kawakami, Y.; Kiyono, T.; Yonemura, S.; Kawamura, Y.; Era, S.; Matsuzaki, F.; Goshima, N.; Inagaki, M. Ubiquitin-proteasome system controls ciliogenesis at the initial step of axoneme extension. Nat. Commun. 2014, 5, 5081.

46. Villumsen, B.H.; Danielsen, J.R.; Povlsen, L.; Sylvestersen, K.B.; Merdes, A.; Beli, P.; Yang, Y.G.; Choudhary, C.; Nielsen, M.L.; Mailand, N.; et al. A new cellular stress response that triggers centriolar satellite reorganization and ciliogenesis. EMBO J. 2013, 32, 3029-3040.

47. Patil, M.; Pabla, N.; Huang, S.; Dong, Z. Nek1 phosphorylates Von Hippel-Lindau tumor suppressor to promote its proteasomal degradation and ciliary destabilization. Cell Cycle 2013, 12, 166-171.

48. Eguether, T.; Ermolaeva, M.A.; Zhao, Y.; Bonnet, M.C.; Jain, A.; Pasparakis, M.; Courtois, G.; Tassin, A.M. The deubiquitinating enzyme CYLD controls apical docking of basal bodies in ciliated epithelial cells. Nat. Commun. 2014, 5, 4585.

(C) 2015 by the authors; licensee MDPI, Basel, Switzerland. This article is an open access article distributed under the terms and conditions of the Creative Commons Attribution license (http://creativecommons.org/licenses/by/4.0/). 